Full length article

\title{
Strong optical nonlinearity of ultrathin graphitic films synthesized on dielectric substrates
}

\author{
Tommi Kaplas $^{\mathrm{a}, \mathrm{b}, *, 1}$, Masoud Babaeian ${ }^{\mathrm{c}, \mathrm{d}, 1}$, Benjamin Cromey $^{\mathrm{d}}$, Marian Baah ${ }^{\mathrm{a}}$, Petr Obraztsov , $^{\mathrm{e}}$ \\ Farhad Akhoundi $^{\mathrm{d}}$, N. Peyghambarian ${ }^{\mathrm{d}}$, Khanh Kieu ${ }^{\mathrm{d}}$, Yuri Svirko ${ }^{\mathrm{a}}$ \\ ${ }^{a}$ Institute of Photonics, University of Eastern Finland, Yliopistokatu 7, FI-80101 Joensuu, Finland \\ ${ }^{\mathrm{b}}$ Department of Optoelectronics, Center for Physical Sciences and Technology, Saulétekio al. 3, LT-10222 Vilnius, Lithuania \\ ${ }^{\mathrm{c}}$ Department of Physics, University of Arizona, Tucson, AZ 85721, USA \\ ${ }^{\mathrm{d}}$ College of Optical Sciences, University of Arizona, Tucson, AZ 85721, USA \\ e A. M. Prokhorov General Physics Institute, Moscow, 119991, Russia
}

A R T I C L E I N F O

\section{Keywords:}

Graphitic thin films

Transfer-free synthesis

Graphene

Nonlinear optics

Saturable absorption

\begin{abstract}
A B S T R A C T
We propose and demonstrate a scalable technique to grow a thin polycrystalline graphitic film directly onto a fused silica substrate. The technique is based on the pyrolysis of a photoresist in the presence of a sacrificial $10 \mathrm{~nm}$ thick nickel catalyst layer. The synthesized graphitic film with a thickness of about $50 \mathrm{~nm}$ possesses almost constant $40 \%$ absorptance over visual and near infrared spectral regions. By using Raman characterization, third harmonic generation spectroscopy, and the $Z$-scan technique we perform a comparative study of the films pyrolyzed with and without a Ni catalyst. We show that the amorphous carbon dominates the linear and nonlinear optical properties of the resist film pyrolyzed without the Ni catalyst. In contrast, in presence of a $\mathrm{Ni}$ catalyst layer, the pyrolysis leads to a graphitic film that demonstrates a strong saturable absorption behavior at $1550 \mathrm{~nm}$ wavelength and has a nonlinear refractive index comparable with that of graphene. Thus, the developed, transfer-free synthesis technique provides an alternative route towards the controllable growth of wafer scale graphitic films on the dielectric substrates for photonics applications.
\end{abstract}

\section{Introduction}

Dispersion-less absorption, strong optical nonlinearity and pronounced photon drag effect [1-4] make single- and few-layer graphene as well as ultrathin graphitic films an appealing platform for next generation photonic devices [1-10]. However, chemical vapor deposition (CVD), which has become a conventional synthesis technique of the $\mathrm{sp}^{2}$-hybridized carbon films [11-13], requires catalytic transitional metal substrates (e.g. copper or nickel). Thus, in order to use the synthesized films in electronics and photonics, to the film must be transferedonto dielectric or semiconductor substrates. Although, in recent years some progress has been made [14], this task still remains rather challenging especially because it is needed to be implemented on the industrial scale. Correspondingly, a scalable deposition of highly crystalline $\mathrm{sp}^{2}$-hybridized carbon films onto dielectric and semiconductor support is eagerly awaited because it will open avenues towards fabrication of graphene-based photonic and optoelectronic devices $[2,15,16]$.
In contrast to monolayer CVD graphene, $\mathrm{sp}^{2}$-hybridized polycrystalline graphitic films, which can also be a versatile material for optics and photonics [10], have been rather sparsely studied. Synthesis of thin graphitic films comprised of tiny graphite crystallites (graphene flakes) directly on a dielectric support has been demonstrated by using sacrificial nanometrically layers of metal catalyst [17-22]. Although the grainy structure of these films greatly suppresses the charge carriers mobility, thus hampering electronic applications [18,20,21], their optical properties in the visual and near-IR range resemble those of conventional CVD graphene [17-23]. This makes direct deposition of such films attractive for the development of carbon-based linear and nonlinear optical components [2].

Due to its high carbon solubility, bulk nickel substrates are conventionally used for synthesis of multi-layer graphene [12]. However, it has been demonstrated that graphene and graphitic thin films can be grown from the solid precursor deposited of a nanometrically thin nickel film on dielectric surface [22]. Although such an approach provides a plausible pathway towards synthesis of graphitic films on

\footnotetext{
* Corresponding author at: Institute of Photonics, University of Eastern Finland, Yliopistokatu 7, FI-80101 Joensuu, Finland.

E-mail address: tommi.kaplas@uef.fi (T. Kaplas).

${ }^{1}$ These authors contributed equally.
} 
various substrates, the optical properties of such directly deposited films remain virtually unexplored.

In this paper, we compare the optical properties of photoresist film, which was pyrolyzed with and without nanometrically thin nickel catalyst. We demonstrate that a photoresist, which is deposited on the bare silica surface produces an ultrathin amorphous carbon film after pyrolysis. Additionally, we show that pyrolysis of the photoresist film deposited on the top of the sacrificial $10 \mathrm{~nm}$ thick nickel layer produces polycrystalline graphitic film with a much stronger optical nonlinearity. This amorphous pyrolyzed photoresist film (PPF) can be transformed into a graphitic PPF (GrPPF), which possesses improved - in comparison with the PPF - crystallinity. This advance can be very useful, for instance, in graphene enhanced Raman spectroscopy [22] or in optical computing [24]. It is worth noting that although the presented technique utilizes photoresist as a carbon precursor, we believe that other polymer materials [27] or pyrolytic carbon $[25,26]$ may also be considered as precursors.

\section{Experimental}

\subsection{Sample preparation}

We synthetized an amorphous pyrolyzed photoresist film (PPF) and a graphitic PPF (GrPPF) on the same fused silica substrate using the CVD process. A schematic of the sample fabrication is shown in Fig. 1. The $10 \mathrm{~nm}$ thick Ni film was evaporated on the half of the silica wafer (diameter of $50 \mathrm{~mm}$ ) after which the whole substrate was spin coated with $350 \mathrm{~nm}$ thick nLOF resist layer, soft baked at $110^{\circ} \mathrm{C}$ for $1 \mathrm{~min}$. The resist was pyrolyzed at temperature of $800^{\circ} \mathrm{C}$ for $10 \mathrm{~min}$ in an $\mathrm{H}_{2}$ atmosphere ( $1 \mathrm{mBar}$ ) by using a conventional hot wall chemical vapor deposition (CVD) reactor. After the process, the CVD reactor was cooled down to room temperature for $12 \mathrm{~h}$ in a static $\mathrm{H}_{2}$ atmosphere (5 mBar).

At $800^{\circ} \mathrm{C}$, the resist film was pyrolyzed, while surface melting of the ultrathin Ni film resulted in the formation of micron and sub-micron size Ni particles. The particles were partially covered with a thin layer of the pyrolyzed resist, which was removed by a short $(100 \mathrm{~W}, 20 \mathrm{sccm}$, $10 \mathrm{~s}$ ) oxygen plasma treatment. The Ni remains were then removed by wet etching $\left(\mathrm{CuSO}_{4}-\mathrm{HCl}-\mathrm{H}_{2} \mathrm{O}\right.$ solution) and the sample was rinsed in water. When the Ni particles were removed, the thickness of the pyrolyzed resist film was about $60 \mathrm{~nm} \pm 5 \mathrm{~nm}$, while the thickness of the resist that was pyrolyzed without $\mathrm{Ni}$ was $50 \mathrm{~nm} \pm 2 \mathrm{~nm}$.

\subsection{Characterization}

Scanning electron microscopy (SEM) characterization of the pyrolyzed film in the vicinity of the Ni film edge (Fig. 2 a) revealed that $\mathrm{Ni}$ particles were removed from the sample and that the resist film pyrolyzed in presence of nickel had micron- and submicron size holes that correspond to the removed Ni particles [22]. By high resolution SEM characterization we confirmed that $\mathrm{Ni}$ particles were fully removed from the GrPPF during the Ni removal process (see Fig. S1 supporting material). In contrast, the surface of the resist film pyrolyzed without nickel was uniform throughout the deposited area (Fig. 2 c).

In order to examine the crystallinity of the carbon film, we compared the Raman spectra of the nLOF resist layer pyrolyzed with and without Ni film underneath (Figure, 3a). The Raman spectra shown in Fig. 3b., each measured at different points on the sample shown in Fig. 3a, have dominant $D$ and $G$ peaks at $1350 \mathrm{~cm}^{-1}$ and $1583 \mathrm{~cm}^{-1}$, respectively. The $\mathrm{D}$ peak represents intervalley phonon and defect scattering, showing a degree of disorder in the graphene sheets, while the $\mathrm{G}$ peak is a signature of the primary in-plane graphene vibrational mode [28]. By comparing the magnitudes of the D peaks seen in Fig. 2b, we observed that the D peak was significantly suppressed at points 1,2 and 3 , in comparison to point 4 in an area which was synthetized without Ni (see also Supporting material Fig. S2) Especially, point 1 shows that the D-peak was almost fully removed. This observation was consistent with Fig. 3c, which shows a map of the D peak over the sample surface. Moreover, the $\mathrm{G}$ peak presented in Fig. 3b shows that the full-width-half-maximum (FWHM) was about $28 \mathrm{~cm}^{-1}$ and $100 \mathrm{~cm}^{-1}$ for the resist layer pyrolyzed with and without nickel, respectively. The suppressed D peak and the narrower G peak both indicate that the level of disorder in the film pyrolyzed without nickel was much higher than that in the film pyrolyzed in the presence of the $\mathrm{Ni}$ droplets [28-30]. The latter conclusion was supported by mapping the 2D peak at $2700 \mathrm{~cm}^{-1}$ (Fig. 3e), the second order overtone of the Dmode, which is a signature of a graphitic electron band structure [28]. Raman maps for D-, G- and 2D-peaks, shown in Fig. 3c, d and e, respectively, corresponded to the area restricted with a dashed line in Fig. 3a. In summary, Raman characterization shows that PPF synthetized with nickel has many similarities to graphene and graphite, while the PPF synthetized without nickel has an amorphous nature [28-30].

Another experimental sign of the graphitic nature of the GrPPF film was the almost constant optical absorption in the spectral range between 400 and $1800 \mathrm{~nm}$ [1], measured by a spectrophotometer (see details in Supporting material). The spectra in Fig. 2f-h demonstrates nearly constant values for transmittance, reflectance and absorptance (40\%, 20\% and 40\%, respectively). Furthermore, the GrPPF showed a clear absorption peak at $260 \mathrm{~nm}$, which was similar to the M-saddle point absorption in graphene [31]. Despite the fact that GrPPF contains many defects, being a grainy, polycrystalline material (Fig. 2), its optical properties are similar to crystalline graphite [29,31]. It is worth noting that in contrast to GrPPF, the transmittance, reflectance and absorptance of PPF show a considerable wavelength dependence in the $400-1800 \mathrm{~nm}$ range, indicating an absence of the graphitic electron band structure.

\subsubsection{Nonlinear optical characterization}

To visualize the difference in the optical nonlinearities of GrPPF and $\mathrm{PPF}$, we probed the surface of the pyrolyzed carbon film with a scanning multiphoton microscope with a lateral resolution of a few microns (detailed description of the microscope can be found in [23,32]). In the setup shown in Fig. 4a (inset), a laser with a FWHM pulse duration of $200 \mathrm{fs}$, a center wavelength of $1550 \mathrm{~nm}$, and a repetition rate of $8 \mathrm{MHz}$ was used. This fluence of $29 \mathrm{~mJ} / \mathrm{cm}^{2}$ was low enough to avoid damaging the carbon films. Fig. 4a shows spectra of the light generated near the border between GrPPF and PPF obtained by a spectral analyzer. The images shown in Fig. 4b and c illustrate the images of the border area. The signal between 560 and $830 \mathrm{~nm}$ was expected to originate from hot electron fluorescence $[1,31,33]$, while the signal in the range of $507-520 \mathrm{~nm}$ corresponded to third harmonic generation (THG).

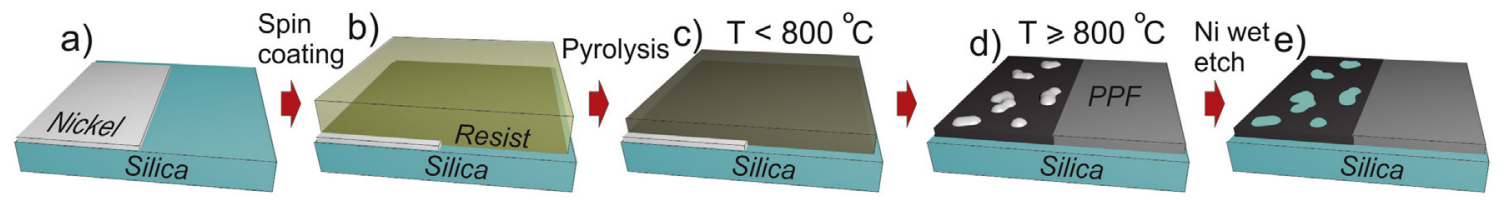

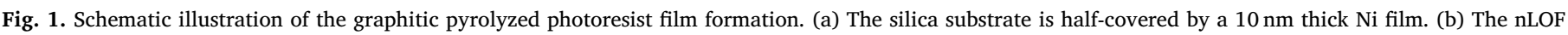

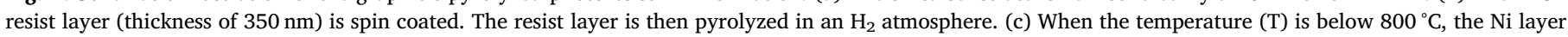

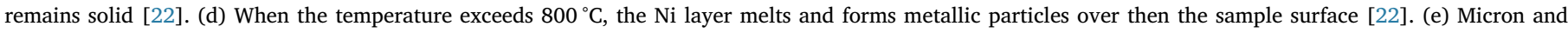
submicron size holes remain in the graphitic film after removing the melted Ni residues by the wet etching. 


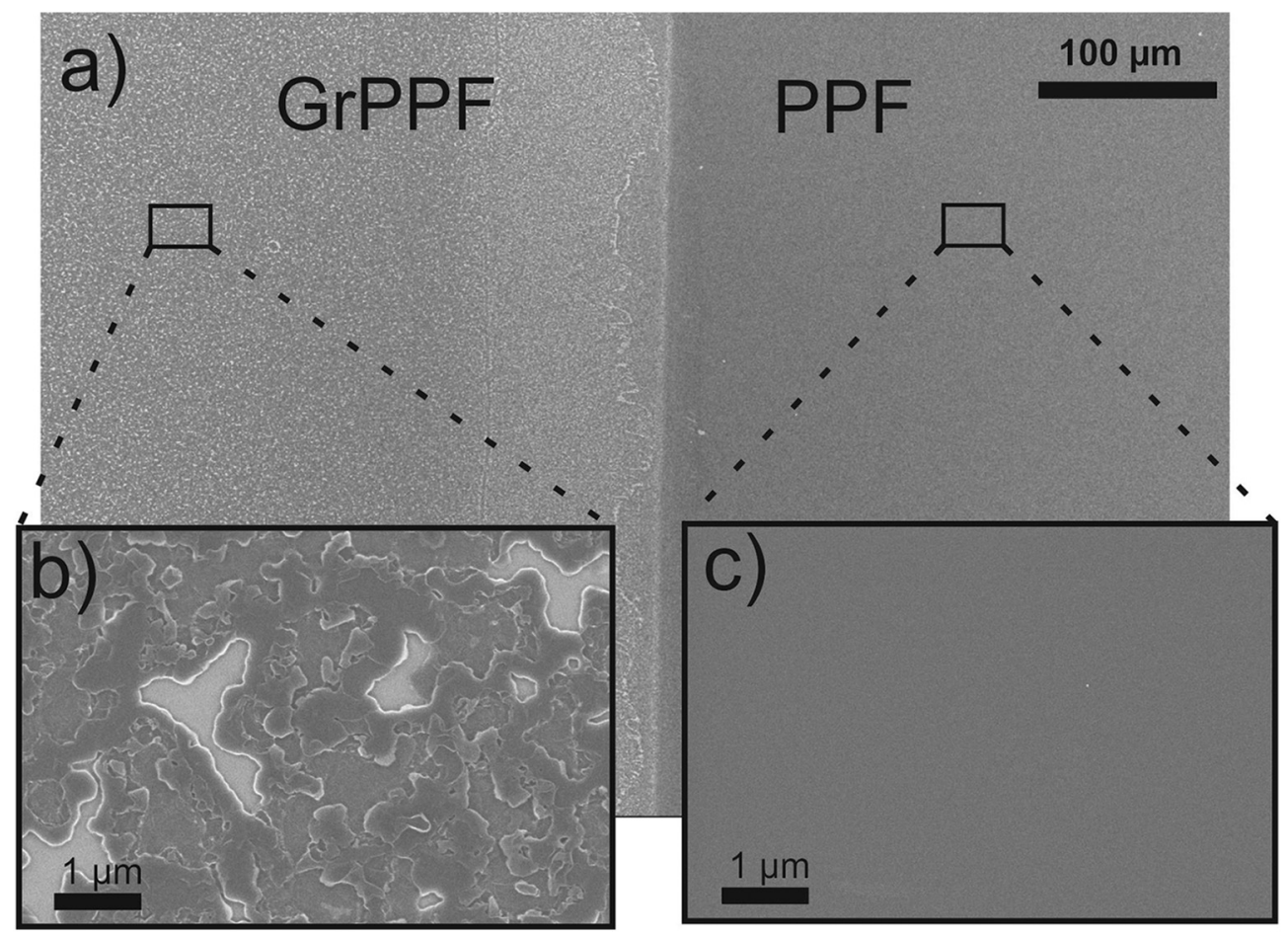

Fig. 2. (a) SEM image of the pyrolyzed resist in the vicinity of the Ni film edge. (b) The film pyrolyzed in the presence of the Ni film (left part of the image) was filled with holes due to the etched out melted Ni particles [22]. (c) The pyrolyzed film was uniform in the area with no Ni [22,25].
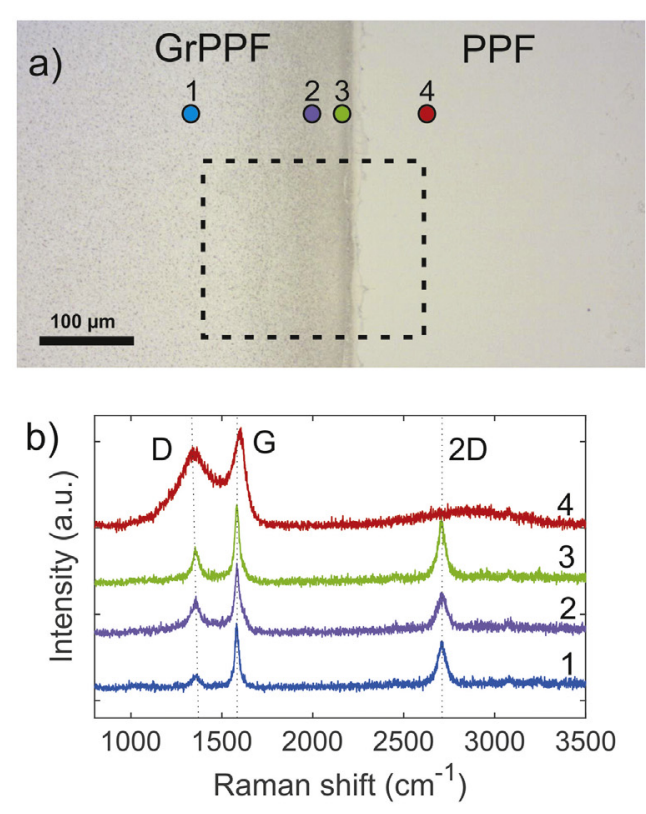

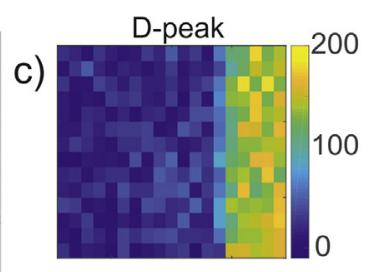

d)
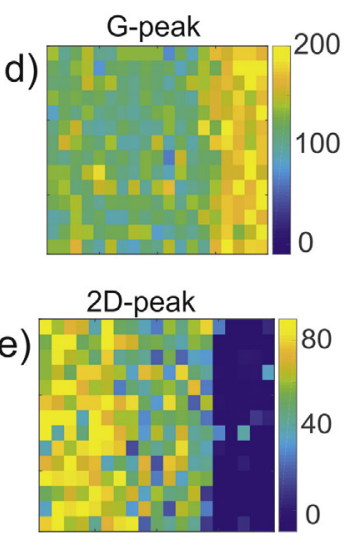
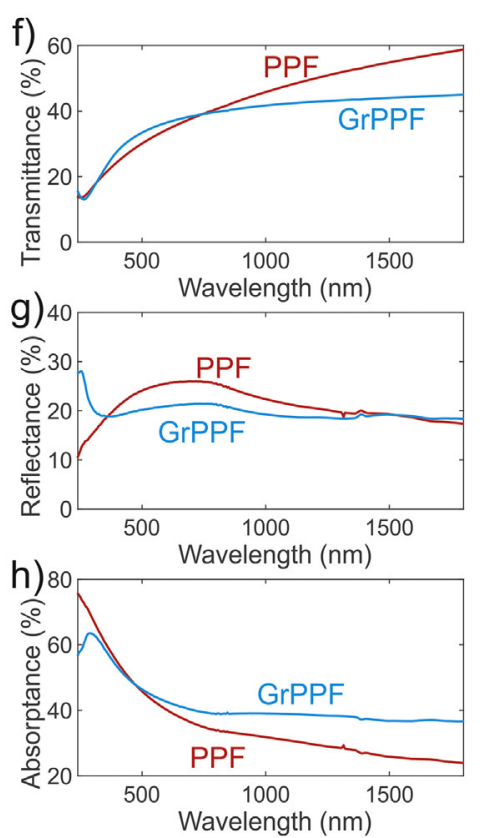

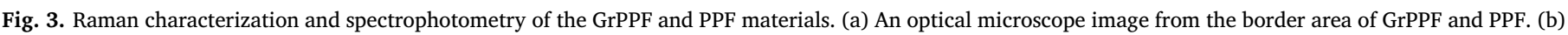

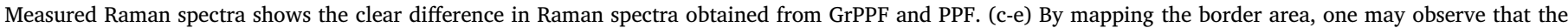

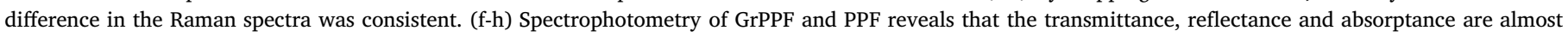
constant for GrPPF at visual and near infrared spectral region, while for PPF these values depend on wavelength.

The observed PL signal from the GrPPF was about 12 times stronger than that of PPF (see Fig. 4b and Supporting information), while THG signal from GrPPF was just 2.5 times stronger in comparison to that of PPF. Since the thicknesses of both films are almost the same, one may conclude that third-order electronic nonlinear susceptibility of GrPPF was approximately 1.6 times higher that of PPF.

In addition, we performed Z-scan measurements with an open aperture $(\mathrm{OA})$ and closed aperture (CA) setup to further examine the nonlinear properties [34,35]. In the Z-scan experiment, the FWHM pulse duration was $187 \mathrm{fs}$, with a central wavelength at $1550 \mathrm{~nm}$ and a pulse repetition rate of $50 \mathrm{~Hz}$. Because of the low repetition rate, the thermal effects in carbon films were suppressed, allowing the use of high intensity pulses without damaging the carbon films. The damage threshold of GrPPF and PPF was observed to be around $140 \mathrm{GW} / \mathrm{cm}^{2}$ and $190 \mathrm{GW} / \mathrm{cm}^{2}$, respectively.

In the open aperture Z-scan measurements, the GrPPF showed 

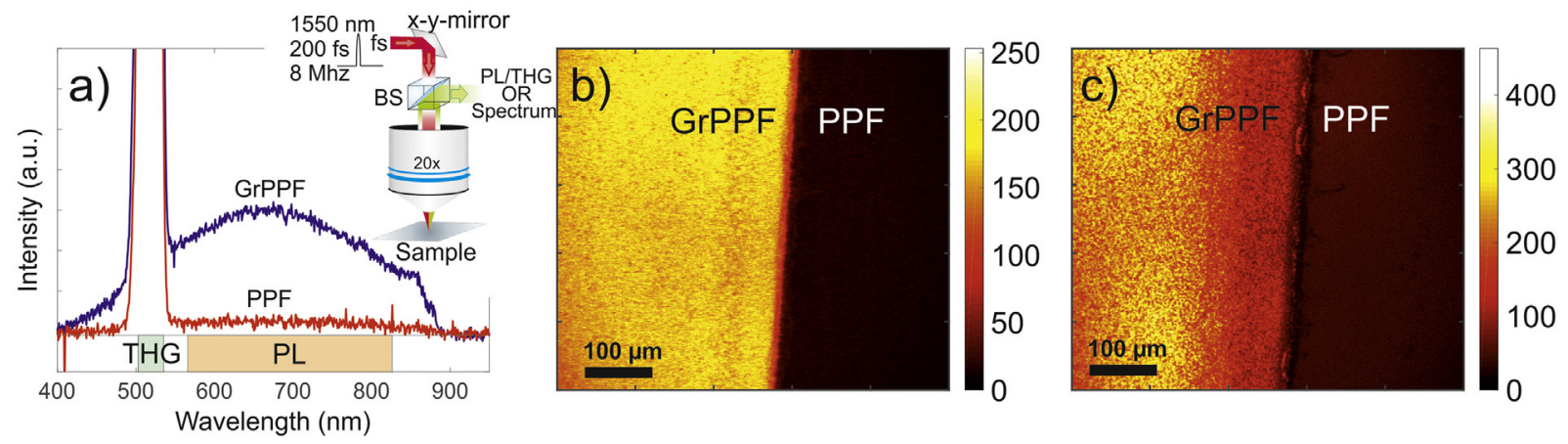

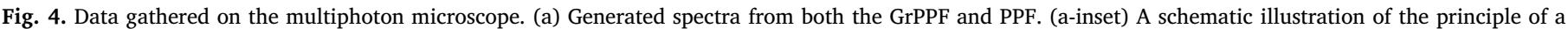

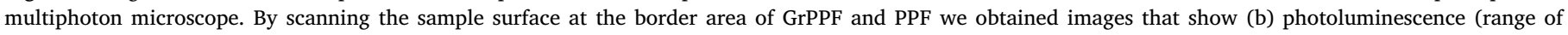
$560 \mathrm{~nm}-830 \mathrm{~nm}$ ) and (c) THG image (range of $507 \mathrm{~nm}-527 \mathrm{~nm}$ ). Color bar in (b) and (c) denotes the intensity of generated signals.
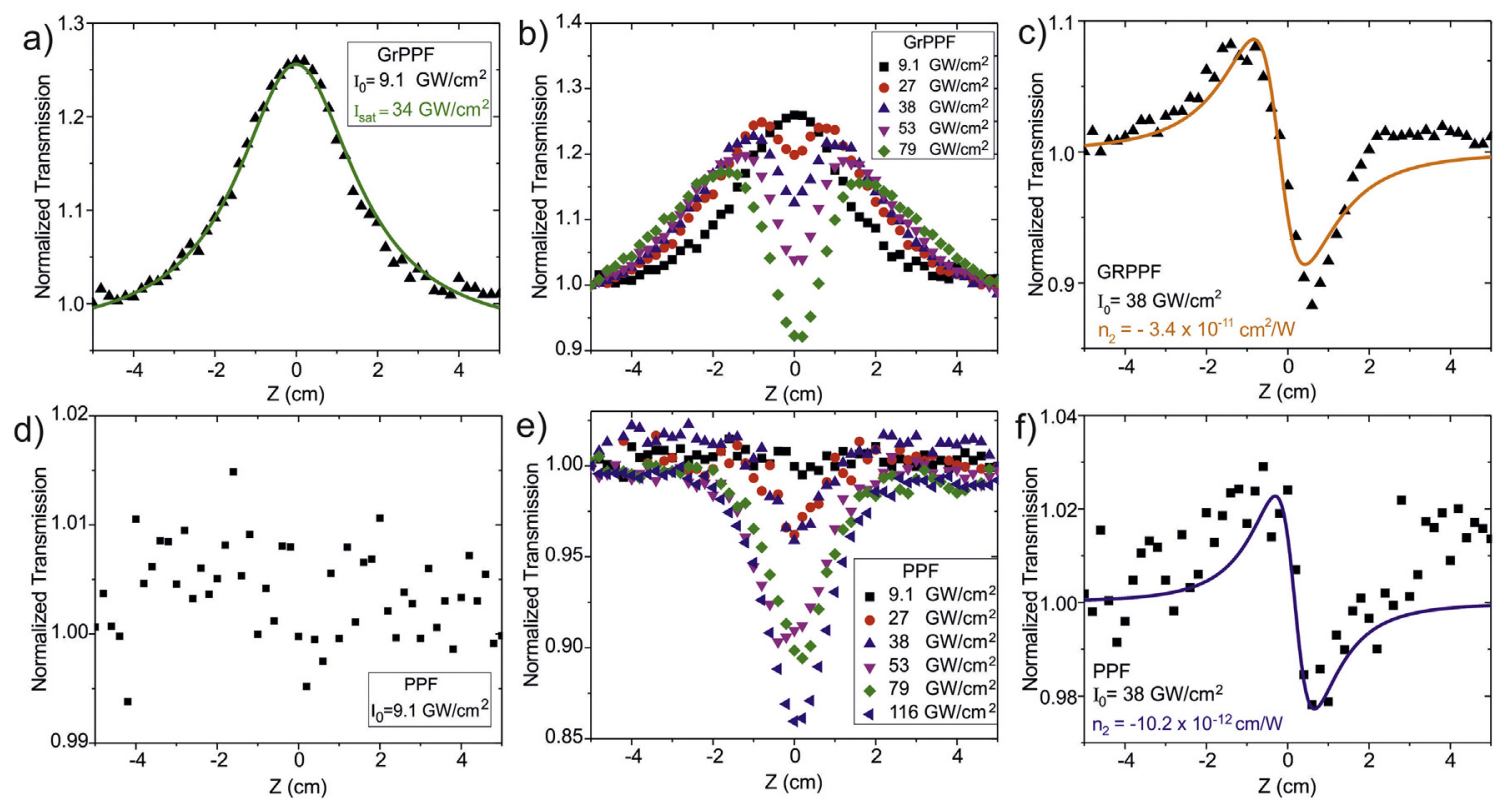

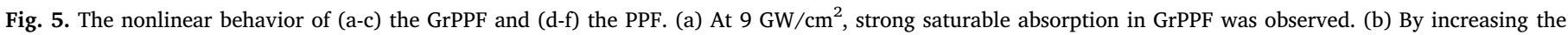

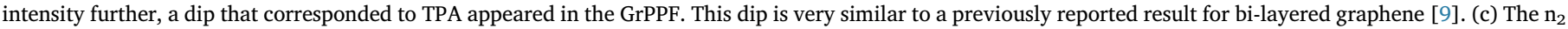

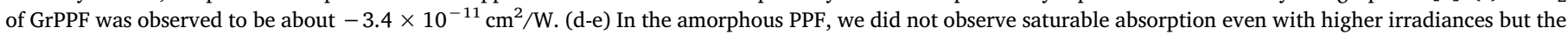
TPA signal was clearly observable. (f) The $\mathrm{n}_{2}$ of the PPF was about $-10.2 \times 10^{-12} \mathrm{~cm}^{2} / \mathrm{W}$.

saturable absorption (SA) behavior (Fig. 5a) at the lowest measured peak irradiance of $9.1 \mathrm{GW} / \mathrm{cm}^{2}$. Fig. 5a shows the normalized transmission for the OA setup as a function of the sample position and its fit with the SA model.

In the framework of the SA model, the evolution of the beam intensity $I$ inside nonlinear medium is described by the following equation [36].

$\frac{d I}{d z^{\prime}}=-\frac{\alpha_{0}}{1+I / I_{\text {sat }}} I$,

where $I_{s a t}$ is the saturation intensity, $z^{\prime}$ is the propagation distance inside the sample, and $\alpha_{0}$ is the linear absorption coefficient. The incident peak irradiance at the front surface of the sample $I\left(z^{\prime}=0\right)$ is determined by the sample position $z$ with respect of the focal point:

$I\left(z^{\prime}=0\right)=\frac{I_{0}}{1+z^{2} / z_{R}^{2}}$,

where $I_{0}$ is the peak irradiance at the focal point and $z_{R}$ is the Rayleigh length, which was much longer than the sample thickness of $50 \mathrm{~nm}$. The experimental data in Fig. 5a was fitted with a numerical solution to Eq. (1) (green solid line) and the $I_{\text {sat }}$ and $\alpha_{0}$ were estimated to be $34 \mathrm{GW} /$ $\mathrm{cm}^{2}$ and $46.8 \times 10^{3} \mathrm{~cm}^{-1}$, respectively (for further details see the
Supporting information). For the higher input irradiances, we observed a dip which could correspond to two-photon absorption (TPA), similar to that of in bi-layered graphene [9].

It is clear from Fig. $5 \mathrm{~d}$ that the PPF sample did not show SA behavior in the $Z$-scan experiment. This was in line with our earlier measurements with an amorphous pyrolytic carbon film [37]. However, the PPF showed strong TPA at elevated irradiance. In the presence of the TPA the evolution of the beam intensity inside the sample can be described by the following equation [38]:

$\frac{d I}{d z^{\prime}}=-\alpha_{0} I-\beta I^{2}$,

where $\alpha_{0}$ and $\beta$ are linear and two-photon absorption coefficients. For the incident beam intensity given by Eq. (2) the normalized peak irradiance transmittance $T$ is given by [34,35].

$T=\sum_{m=0}^{\infty} \frac{\left[-q_{0}(z)\right]^{m}}{(m+1)^{3 / 2}}$

where, $q_{0}(z)=\beta I_{0} L_{e f f} /\left(1+z^{2} / z_{R}^{2}\right), L_{e f f}=\left(1-e^{\alpha_{0} L}\right) / \alpha_{0}$, and $L$ is the thickness of the sample. The linear absorption coefficient at $1550 \mathrm{~nm}$ was measured to be $\alpha_{0} \sim 40.0 \times 10^{3} \mathrm{~cm}^{-1}$. Fig. $5 \mathrm{f}$ shows the normalized 
transmission of the PPF as a function of sample position taken at different irradiances for the OA configuration. The fitting with Eq. (4) returns $\beta \sim(970 \pm 50) \mathrm{cm} / \mathrm{GW}$ for the PPF.

The nonlinear refractive index of the GrPPF was extracted from the $\mathrm{CA}(\mathrm{S} \sim 0.36) \mathrm{Z}$-scan data and fitted with the following equation for the normalized transmittance [34,35].

$T=1-\frac{4 x \Delta \varphi_{0}}{\left(x^{2}+9\right)\left(x^{2}+1\right)}$,

where $x=z / z_{R}$, and $\Delta \varphi_{0}=2 \pi I_{0} L_{e f f} n_{2} / \lambda$ is the nonlinear phase shift at the exit surface of the sample, which is proportional to the nonlinear refractive index $n_{2}$.

Subtracting the transmission data of the SA behavior from the CA transmission data, we can determine the nonlinear refractive index for GrPPF by fitting the normalized transmission data for the closed aperture with Eq. (5). For the GrPPF and the PPF, the obtained nonlinear refraction index was $-(3.4 \pm 0.2) \times 10^{-11} \mathrm{~cm}^{2} / \mathrm{W}$ and $-(10.2 \pm 0.5) \times 10^{-12} \mathrm{~cm}^{2} / \mathrm{W}$, respectively. Therefore, the $\mathrm{n}_{2}$ absolute value for GrPPF was approximately 3.5 times larger than that for the PPF.

\section{Discussion}

The clear D- and G-peaks of Raman spectra for GrPPF and PPF (Fig. 3b) indicate that both carbon films have dominant $\mathrm{sp}^{2}$ hybridization [29]. However, the measured D- and G-peaks of the PPF were broad and the ratio of D- and G-peaks was close to one, indicating the carbon material was very amorphous $[29,30]$. In GrPPF, the D- and G-peaks are sharp, and the D-peak was significantly suppressed indicating a high degree of crystallization [29]. The GrPPF Raman spectrum shows a strong 2D peak at $2700 \mathrm{~cm}^{-1}$, which was not observed in the PPF sample. Since the 2D resonance mode was recognized as a signature of graphitic structure [28], the results of our Raman measurements implied that polycrystalline GrPPF possessed a graphitic electron band structure. It is also worth noticing that the position of the $\mathrm{G}$ peak was located at $1583 \mathrm{~cm}^{-1}$, which indicates very low doping levels in the GrPPF [28]. Due to low doping, the location of 2D peak at $2700 \mathrm{~cm}^{-1}$ was explained by multilayered graphene stacking, which shifts 2D peak from $\sim 2685 \mathrm{~cm}^{-1}$ (monolayer graphene) towards $2730 \mathrm{~cm}^{-1}$ (bulk graphite) [28,29].

The IR transmittance, reflectance, and absorptance spectra in Fig. 3f, $g$ and h, respectively, show that the linear absorption coefficient of the GrPPF was almost constant, while that of PPF was not. In the IR range, the absorption in graphitic materials is governed by $\pi-\pi^{*}$ electron transitions $[1,31]$. Due to the linear electron band structure in a graphitic material, the $\pi$-electron transitions give rise to frequency independent absorption in the near-IR photon energy range [31]. Since the IR absorptance of GrPPF was almost dispersion-less, one may assume that it possessed a linear electron band structure similar to that of graphene [1]. In contrast, the PPF absorbance showed a pronounced wavelength dependence, which indicated that it was very different from graphene band structure.

Absorption spectra of graphene [31] and GrPPF showed a resonance at about $260 \mathrm{~nm}$, which is associated with the M-saddle point absorption. This resonance was not seen in the PPF absorptance spectrum (see Fig. 3h), but the absorptance did increase in ultra-violet range due to the presence of aromatic carbon structures [39]. It is worth noting that M-saddle point absorption resonance can be observed in PPF pyrolyzed at $1100{ }^{\circ} \mathrm{C}$ [25].

The graphene-like band structure of the GrPPF implied that the observed broad PL spectrum shown in Fig. 3a originated from hot electron emission [1,33]. It has been suggested that in graphene, a photoluminescence spectrum can be generated by optical pumping $[1,33]$. This broadband photoluminescence was created by photothermalized electron-hole scattering [33]. In the amorphous PPF, we observed a very faint PL signal in comparison to GrPPF. Even though the PL signal was more than one order of magnitude smaller in the PPF, the signal was still present in both materials (see Supporting information). This weak PL may have originated from aromatic carbon structures in the PPF.

Furthermore, from the multiphoton microscope image (Fig. 4c), we concluded that in the GrPPF, the THG signal was approximately 2.5 times stronger than that from the PPF. Since the THG intensity $I$ $(3 \omega) \propto\left|\chi^{(3)}\right|^{2}[I(\omega)]^{3}$ was determined by the third-order susceptibility $\chi^{(3)}$, we calculated that $\left|\chi^{(3)}\right|$ of the GrPPF was about 1.6 times higher than that of the PPF. Moreover, CA $Z$-scan measurements show the nonlinear refractive index $n_{2}$ of the GrPPF, which is proportional to $R e$ $\left\{\chi^{(3)}\right\}$, was approximately 3.5 times higher in magnitude compared to that of the amorphous PPF.

In the GrPPF, the $Z$-scan measurements revealed saturable nonlinear absorption, which is important for practical applications of graphene. The absorption saturation can be described (at $I<I_{\text {sat }}$ ) in terms of the negative nonlinear absorption coefficient, $\beta_{\text {GrPPF }} \approx-\frac{\alpha_{0}}{I_{\text {sat }}} \approx-1400 \mathrm{~cm} /$ GW. The nonlinear absorption in the PPF was governed by the twophoton process, indicating that the nonlinear absorption coefficient was positive, $\beta_{P P F} \approx 970 \mathrm{~cm} / \mathrm{GW}$. However, the ratio $\left|\beta_{G r P P F}\right| / \beta_{P P F} \approx 1.44$ was close to the value obtained from the THG experiment ratio of the nonlinear susceptibilities, $\left|\chi_{G r P P F}{ }^{(3)} / \chi_{P P F}{ }^{(3)}\right| \approx 1.6$. The multiphoton microscope uses a high repetition laser as a probe. Therefore, the small difference in these values, obtained with different setups, can originate from thermal effects, which may affect linear optical properties of the material. However, the good correspondence indicates that that electronic mechanism of the nonlinearity governed both THG and nonlinear absorption in both materials.

Reported values for $\mathrm{n}_{2}$ of graphene vary from $10^{-7}$ to $10^{-13} \mathrm{~cm}^{2} / \mathrm{W}$ depending on the measurement technique and wavelength $[7,8,40]$. This can be partially because of a typically low signal-to-noise ratio in the experiments with atomically thin films. In contrast the to Z-scan measurements with graphene, the optical density of the GrPPF and PPF was higher, resulting in better signal to noise ratio. The obtained $\mathrm{n}_{2}$ for GrPPF and PPF was on the order of $\sim 10^{-11} \mathrm{~cm}^{2} / \mathrm{W}$. This was less than two orders of magnitude lower than measurements from a high quality, transferred, few-layer graphene (5-7 layers) [8], was comparable to that of graphene dispersions, [41] and was about six orders of magnitude higher than that of common dielectrics. Furthermore, the obtained $I_{\text {sat }}$ value from GrPPF was one order of magnitude higher than that of few- and multilayer graphene (which have thickness less than $10 \mathrm{~nm}$ ) $[8,42]$. In [42], it was shown that $I_{s a t}$ is directly proportional to the number of graphene layers. This partially explains the high $I_{\text {sat }}$ we obtained in the GrPPF, which has a thickness of about 5-20 times higher than that in $[8,42]$. Moreover, $I_{\text {sat }}$.is inversely proportional to $\alpha_{0}$, which was suppressed by the presence of the micron size holes in the GrPPF.

\section{Conclusion}

We demonstrate that a nanometrically thin nickel catalyst layer can have a tremendous effect on the crystallinity and optical behavior of a thin carbon film. Furthermore, the developed technique offers an interesting playground for different experimental studies. For instance, tuning the substrate surface could give control over the melted nickel particles, similar to what was earlier reported with copper [43,44]. Also combining different catalysts layers and substrate materials in the same substrate (e.g. nanometrically thin $\mathrm{Cu}[18,19]$ and Ni layers on sapphire substrate $[45,46])$, can provide interesting, new approaches for graphitic thin film synthesis. In addition to synthesis techniques, our study shows that polycrystalline graphitic carbon film can perform very well in optics. Due to its constant absorption over a wide spectrum range, saturable absorption, high damage threshold and relatively high $\mathrm{n}_{2}$, the GrPPF could be a practical graphene alternative for photonic applications such as $\mathrm{THz}$ detectors [47]. 


\section{Acknowledgements}

This work was financially supported by Academy of Finland (grants nos. 287886, 318596 and 298298), EU H2020 Project no. 823728 DiSetCom, Russian Science Foundation (grant no.17-72-10303), NPNano Fidipro-project by the Finnish Funding Agency for Innovation (TEKES) and Väisälä Foundation.

\section{Appendix A. Supplementary data}

Supplementary data to this article can be found online at https:// doi.org/10.1016/j.apsusc.2019.143766.

\section{References}

[1] F. Bonaccorso, T.H.Z. Sun, A.C. Ferrari, Graphene photonics and optoelectronics, Nature Phot 4 (2010) 611.

[2] K.S. Novoselov, V.I. Falko, L. Colombo, P.R. Gellert, M.G. Schwab, K. Kim, A roadmap for graphene, Nature 490 (2012) 192.

[3] R.R. Nair, P. Blake, A.N. Grigorenko, K.S. Novoselov, T.J. Booth, T. Stauber, et al., Fine structure constant defines visual transparency of graphene, Science 320 (2008) 1308.

[4] P.A. Obraztsov, T. Kaplas, S.V. Garnov, M. Kuwata-Gonokami, A.N. Obraztsov, Y.P. Svirko, All-optical control of ultrafast photocurrents in unbiased graphene, Sci. Rep. 4 (2014) 4007.

[5] E. Hendry, P.J. Hale, J. Moger, A.K. Savchenko, S.A. Mikhailov, Coherent nonlinear optical response of graphene, Phys. Rev. Lett. 105 (2010) 097401.

[6] A. Martinez, K. Fuse, S. Yamashita, Mechanical exfoliation of graphene for the passive mode-locking of fiber lasers, Appl. Phys. Lett. 99 (2011) 121107.

[7] H. Zhang, S. Virally, Q. Bao, L.K. Ping, S. Massar, N. Godbout, et al., Z-scan measurement of the nonlinear refractive index of graphene, Opt. Lett. 37 (2012) $1856-1858$

[8] G. Demetriou, H.T. Bookey, F. Biancalana, E. Abraham, Y. Wang, W. Ji, et al., Nonlinear optical properties of multilayer graphene in the infrared, Opt. Express 24 (2016) 13033.

[9] H. Yang, X. Feng, Q. Wang, H. Huang, W. Chen, A.T.S. Wee, et al., Giant two-photon absorption in bilayer graphene, Nano Lett. 11 (2011) 2622.

[10] R. Podila, B. Anand, J.T. Spear, P. Puneet, R. Philip, S.S.S. Sai, et al., Effects of disorder on the optical properties of CVD grown polycrystalline graphene, Nanoscale 4 (2012) 1770-1775.

[11] C. Mattevi, H. Kim, M. Chhowalla, A review of chemical vapour deposition of graphene on copper, J. Mater. Chem. 21 (2011) 3324.

[12] A. Reina, X. Jia, J. Ho, D. Nezich, H. Son, V. Bulovic, et al., Large area, few-layer graphene films on arbitrary substrates by chemical vapor deposition, Nano Lett. 9 (1) (2009) 30

[13] T. Kaplas, P. Kuzhir, Ultra-thin graphitic film: synthesis and physical properties, Nanoscale Res. Lett. 11 (54) (2016) 1-6.

[14] Y. Zhu, H. Ji, H.-M. Cheng, R.S. Ruoff, Mass production and industrial applications of graphene materials, Natl. Sci. Rev. 5 (1) (2018) 90.

[15] J. Ning, D. Wang, Y. Chai, X. Feng, M. Mu, L. Guo, et al., Review on mechanism of directly fabricating wafer-scale graphene on dielectric substrates by chemical vapor deposition, Nanotechnology 28 (2017) 284001.

[16] G. Kaur, K. Kavitha, I. Lahiri, Transfer-free graphene growth on dielectric substrates: a review of the growth mechanism, Critical Rev. in Solid State and Mat. Sci. (2018), https://doi.org/10.1080/10408436.2018.1433630.

[17] A. Ismach, C. Druzgalski, S. Penwell, A. Schwartzberg, M. Zheng, A. Javey, et al., Direct chemical vapor deposition of graphene on dielectric surfaces, Nano Lett. 10 (5) (2010) 1542.

[18] C.Y. Su, A.Y. Lu, C.Y. Wu, Y.T. Li, K.K. Liu, W. Zhang, et al., Direct formation of wafer scale graphene thin layers on insulating substrates by chemical vapor deposition, Nano Lett. 11 (2011) 3612.

[19] T. Kaplas, D. Sharma, Y. Svirko, Few-layer graphene synthesis on a dielectric substrate, few-layer graphene synthesis on a dielectric substrate, Carbon 50 (4) (2012) 1503.

[20] J. Kwak, J.H. Chu, J.-K. Choi, S.-D. Park, H. Go, S.Y. Kim, et al., Near room-temperature synthesis of transfer-free graphene films, Nature Comm 3 (2012) 645.
[21] D. Wang, H. Tian, Y. Yang, D. Xie, T.-L. Ren, Y. Zhang, Scalable and direct growth of graphene micro ribbons on dielectric substrates, Sci. Rep. 3 (2013) 1348.

[22] T. Kaplas, A. Matikainen, T. Nuutinen, S. Suvanto, P. Vahimaa, Y. Svirko, Scalable fabrication of the graphitic substrates for graphene-enhanced Raman spectroscopy, Sci. Rep. 7 (2017) 8561.

[23] T. Kaplas, L. Karvonen, S. Ahmadi, B. Amirsolaimani, S. Mehravar, N. Peyghambarian, et al., Optical characterization of directly deposited graphene on a dielectric substrate, Opt. Express 24 (2016) 2965-2970.

[24] M. Babaeian, P.-A. Blanche, R.A. Norwood, T. Kaplas, P. Keiffer, Y. Svirko, et al., Nonlinear optical components for all-optical probabilistic graphical model, Nature Comm 9 (1) (2018) 2128.

[25] T. Kaplas, P. Kuzhir, Ultra-thin pyrocarbon films as a versatile coating material, Nanoscale Res. Lett. 12 (1) (2017) 121.

[26] N. McEvoy, N. Peltekis, S. Kumar, E. Rezvani, H. Nolan, G.P. Keeley, et al., Synthesis and analysis of thin conducting pyrolytic carbon films, Carbon 50 (3) (2012) 1216-1226.

[27] T. Ohnishi, I. Murase, T. Noguchi, M. Hirooka, Preparation of graphite film by pyrolysis of polymers, Synth. Met. 18 (1987) 497-502.

[28] A.C. Ferrari, D.M. Basko, Raman spectroscopy as a versatile tool for studying the properties of graphene, Nat. Nanotech. 8 (2013) 235.

[29] T. Jawhari, A. Roid, J. Casado, Raman spectroscopic characterization of some commercially available carbon black materials, Carbon 33 (11) (1995) 1561.

[30] A.C. Ferrari, J. Robertson, Raman spectroscopy of amorphous, nanostructured, diamond-like carbon, and nanodiamond, Phil. Trans. R. Soc. Lond. A 362 (2004) 2477.

[31] K.F. Mak, L. Ju, F. Wang, T.F. Heinz, Optical spectroscopy of graphene: from the far infrared to the ultraviolet, Solid State Comm 152 (2012) 1341.

[32] A. Säynätjoki, L. Karvonen, J. Riikonen, W. Kim, S. Mehravar, R.A. Norwood, et al., Rapid large-area multiphoton microscopy for characterization of graphene, ACS Nano 7 (10) (2013) 8441.

[33] C.H. Lui, K.F. Mak, J. Shan, T.F. Heinz, Ultrafast photoluminescence from graphene, Phys. Rev. Lett. 105 (2010) 127404.

[34] M. Sheik-bahae, A.A. Said, T.H. Wei, D.J. Hagan, E.W. Van Stryland, Sensitive measurements of optical nonlinearities using a single beam, IEEE J. Quantum Electron. 26 (1990) 760.

[35] M. Sheik-bahae, A.A. Said, E.W. Van Stryland, High-sensitivity, single-beam n2 measurements, Opt. Lett. 14 (1989) 9551989.

[36] M. Hercher, An analysis of saturable absorbers, Appl. Opt. 6 (5) (1967) 947.

[37] T. Kaplas, L. Karvonen, J. Rönn, M.R. Saleem, S. Kujala, S. Honkanen, et al., Nonlinear refraction in semitransparent pyrolytic carbon films, Opt. Mat. Express 2 (12) (2012) 1822.

[38] E.W.V. Stryland, H. Vanherzeele, M.A. Woodall, M.J. Soileau, A.L. Smirl, S. Guha, et al., Two photon absorption, nonlinear refraction, and optical limiting in semiconductors, Opt. Eng. 24 (1985) 613.

[39] B. Apicella, M. Alfè, R. Barbella, A. Tregrossi, A. Ciajolo, Aromatic structures of carbonaceous materials and soot inferred by spectroscopic analysis, Carbon 42 (2004) 1583

[40] E. Dremetsika, B. Dlubak, S.-P. Gorza, C. Ciret, M.-B. Martin, S. Hofmann, et al., Measuring the nonlinear refractive index of graphene using the optical Kerr effect method, Opt. Lett. 41 (2016) 3281.

[41] K. Wang, Y. Feng, C. Chang, J. Zhan, C. Wang, Q. Zhao, et al., Broadband ultrafast nonlinear absorption and nonlinear refraction of layered molybdenum dichalcogenide semiconductors, Nanoscale 6 (2014) 10530.

[42] W. Chen, Y. Wang, W. Ji, Two-photon absorption in graphene enhanced by the excitonic fano resonance, J. Phys. Chem. C 119 (2015) 16954.

[43] T. Kaplas, M. Silvennoinen, K. Paivasaari, Y. Svirko, Self-assembled two-dimensional graphene grating on a dielectric substrate, App. Phys. Lett. 102 (21) (2013) 211603

[44] T. Kaplas, Y. Svirko, Self-assembled graphene on dielectric micro-and nanostructures, Carbon 70 (2014) 273

[45] J. Ning, D. Wang, J. Yan, D. Han, Z. Chai, W. Cai, et al., Combined effects of hydrogen annealing on morphological, electrical and structural properties of graphene/r-sapphire, Carbon 75 (2014) 262.

[46] J. Ning, D. Wang, D. Han, Y. Shi, W. Cai, J. Zhang, et al., Comprehensive nucleation mechanisms of quasi-monolayer graphene grown on $\mathrm{Cu}$ by chemical vapor deposition, J. Cryst. Growth 424 (2015) 55.

[47] P. Obraztsov, P. Chizhov, T. Kaplas, V. Bukin, M. Silvennoinen, C.-F. Hsieh, et al., Coherent detection of terahertz radiation with graphene, ACS Photonics 6 (7) (2019) 1780. 